\title{
A VERSATILE LABVIEW-BASED TOOLBOX DESIGN AND MAN-MACHINE INTERFACE FOR THE ELECTRICAL STIMULATION SYSTEM
}

\author{
Ying-Han Chiou ${ }^{1}$, Shih-Ching Chen ${ }^{2,4}$, Jer-Junn LuH ${ }^{3}$, Yasunobu Handa ${ }^{4}$, Jin-Shin Lai ${ }^{5}$, \\ TE-SON KUO ${ }^{1,6}$ \\ ${ }^{1}$ Department of Electrical Engineering, National Taiwan University, \\ ${ }^{2}$ Department of Physical Medicine and Rehabilitation, Taipei Medical University and Hospital, \\ ${ }^{3}$ School and Graduate Institute of Physical Therapy, College of Medicine, National Taiwan \\ University, Taipei, Taiwan \\ ${ }^{4}$ Department of Restorative Neuromuscular Rehabilitation, Graduate School of Medicine, \\ Tohoku University, Sendai, Japan \\ ${ }^{5}$ Department of Physical Medicine and Rehabilitation, College of Medicine, National Taiwan \\ University, \\ ${ }^{6}$ Institute of Biomedical Engineering, College of Medicine and Engineering, National Taiwan \\ University, Taipei, Taiwan
}

\begin{abstract}
This article introduces the modification of a self-developed prototype electrical stimulator. In addition, we describe our new design of a versatile and user-friendly toolbox based on the LabVIEW environment that will enable clinical users and physicians to easily go on to further applications and research. The prototype electrical stimulator is based on the digital signal processor, and the drive stage of the previous model has also been improved by using a modified constant-current circuit. Moreover, we use LabVIEW to implement the man-machine interface and to develop a user-friendly toolbox. This system is versatile and feasible from the viewpoint of the hardware and software designs. With the virtual instrument in the toolbox, the man-machine interface is easy for users to implement and helpful in their further research. Furthermore, this toolbox includes many units and parameters, such as waveform types, currents, stimulation time, and others. The system can be considered a versatile and full-featured stimulator for various applications, with its high flexibility in stimulation patterns and multi-channel designs. The proposed system can produce suitable electrical stimulation by tuning the parameters in the interface. The procedure described above can also be implemented in man-machine interfaces for different research purposes.
\end{abstract}

Biomed Eng Appl Basis Comm, 2006(June); 18: 144-152.

Keywords: electrical stimulator; functional electrical stimulation (FES); LabVIEW; digital signal processor (DSP); man-machine interface (MMI); rehabilitation engineering

Received: Jan. 29, 2005; Accepted: March 30, 2006

Correspondence: Shih-Ching Chen, M.D., Director

Department of Physical Medicine and Rehabilitation, Taipei Medical University and Hospital, No. 252, Wusing

St., Taipei 110, Taiwan

E-mail: csc@tmu.edu.tw 


\section{INTRODUCTION}

Electrical stimulation, which can excite muscle fibers or nerve cells, has been used for many decades as a modality to maintain or restore the muscle activity of paralyzed patients who suffer from neuromuscular disease, spinal cord injuries (SCIs), or related neural impairments. It can be employed in various clinical applications such as preventing muscle atrophy, decreasing muscle spasm, improving local blood circulation and bone growth, relieving pain, cardiac pacing, and functional electrical stimulation (FES) [17]. In addition, the physical conditions of the human body will change after long-term electrical stimulation treatments, for instance, the muscle bulk, strength, and motor response will increase [8-13].

Nowadays, numerous electrical stimulators have been developed, and they demonstrate their feasibility and versatility in different kinds of applications, such as hand function restoration, sit-to-stand training, balance control, and bladder function training [14-25]. However, the functionality design and user-interfaces of most electrical stimulators are usually programmed in $\mathrm{C}$ languages or low-level assembly. This design might be good for engineers who are familiar with computer languages, but, for most physicians and clinical workers, there exists a tremendous gap between the time required to learn $\mathrm{C}$ languages and the time it takes to develop the human-machine interfaces for their own studies. So a high-level programming system would be better than the low-level assembly or $\mathrm{C}$ languages traditionally used for clinical users to design their own human-machine interfaces. If possible, the system should also include graphical user interface (GUI) facilities, which can easily make users familiar with their experiments.

Due to the conventional difficulties of programming GUI for the electrical stimulator, as described above, we have developed a versatile electrical stimulator with a useful and user-friendly toolbox, which is written in a LabVIEW-based environment (National Instruments Inc., USA). LabVIEW is an example of a new form of graphical object-oriented computer language that is easier for physicians and non-professionals to use than the conventional text-based programming languages. In addition, our electrical stimulator demonstrates superiority and versatility when compares with conventional electrical stimulators. Although several electrical stimulators have been developed, their functions are confined to specific applications. They have lacked flexible pattern-generation capability. Moreover, their driving stage couldn't provide highvoltage compliance and linear voltage-to-current conversion at the same time. Our electrical stimulator has the following features: multi-channel stimulator, flexible and programmable waveforms and stimulation patterns, high-voltage compliance and constant-current driving stage, electrical isolation, and a user-friendly toolbox for its man-machine interface. In this paper, we will briefly introduce our electrical stimulator with, we believe, its superiority and versatility, then there will be a step-by-step overview of its innovative userfriendly toolbox for the electrical stimulator.

\section{SYSTEM DESIGN}

\subsection{Hardware Description}

Our electrical stimulation system is based on a digital signal processor (DSP) (TMS320C32, Texas Instruments Inc., USA), which is used to generate the waveforms and stimulation patterns with its highcomputation capabilities [26]. A block diagram of our electrical stimulation system, names National Taiwan University Functional Electrical Stimulator (NTUFES), is found in Fig. 1. There are additional peripherals embedded in the DSP chip, including two timers, one serial port, and two direct-memory-access (DMA) coprocessors. The DSP has 4096 bytes of built-in random-access memory (RAM). It also includes $256 \mathrm{~K}$ bytes of external RAM and $128 \mathrm{~K}$ bytes of flash readonly memory (ROM) for data storage and programming. An 8-bit 4-channel digital-to-analog converter (DAC) (TLC7225, Texas Instruments Inc., USA) is connected to the DSP to generate the waveforms and stimulation patterns. Two 12-bit analog-to-digital converters (ADCs) (AD7862, Analog Device Inc., USA) are mounted in the system for recording the external feedback signals. These converters can be expanded to four channels, respectively, by two analog multiplexers. In addition, the DSP can communicate with the host computer via a universal asynchronized receiver/transmitter (UART) (GM16C550, Hyundai Inc., Korea) and a voltage levelshifting interface (MAX232, Maxim Integrated Products Inc., USA). The $82 \mathrm{C} 55$ chip is also used to implement the general-purpose I/O pins. There are three I/O pins in this chip, so that the keyboard and liquid crystal display (LCD) can be added in the NTUFES system. Furthermore, the NTUFES system can be used in two different areas of clinical practice, i.e., normal clinical usage and development of control strategies in the laboratory, as illustrated in Fig. 2.

In our previous electrical stimulator model, there were some weaknesses in the driving stage design [2627]. To provide high voltage and a constant-current source, the modified Wilson current mirror circuit has been designed. However, half of the current in the transferring resistors is wasted, so efficiency is 


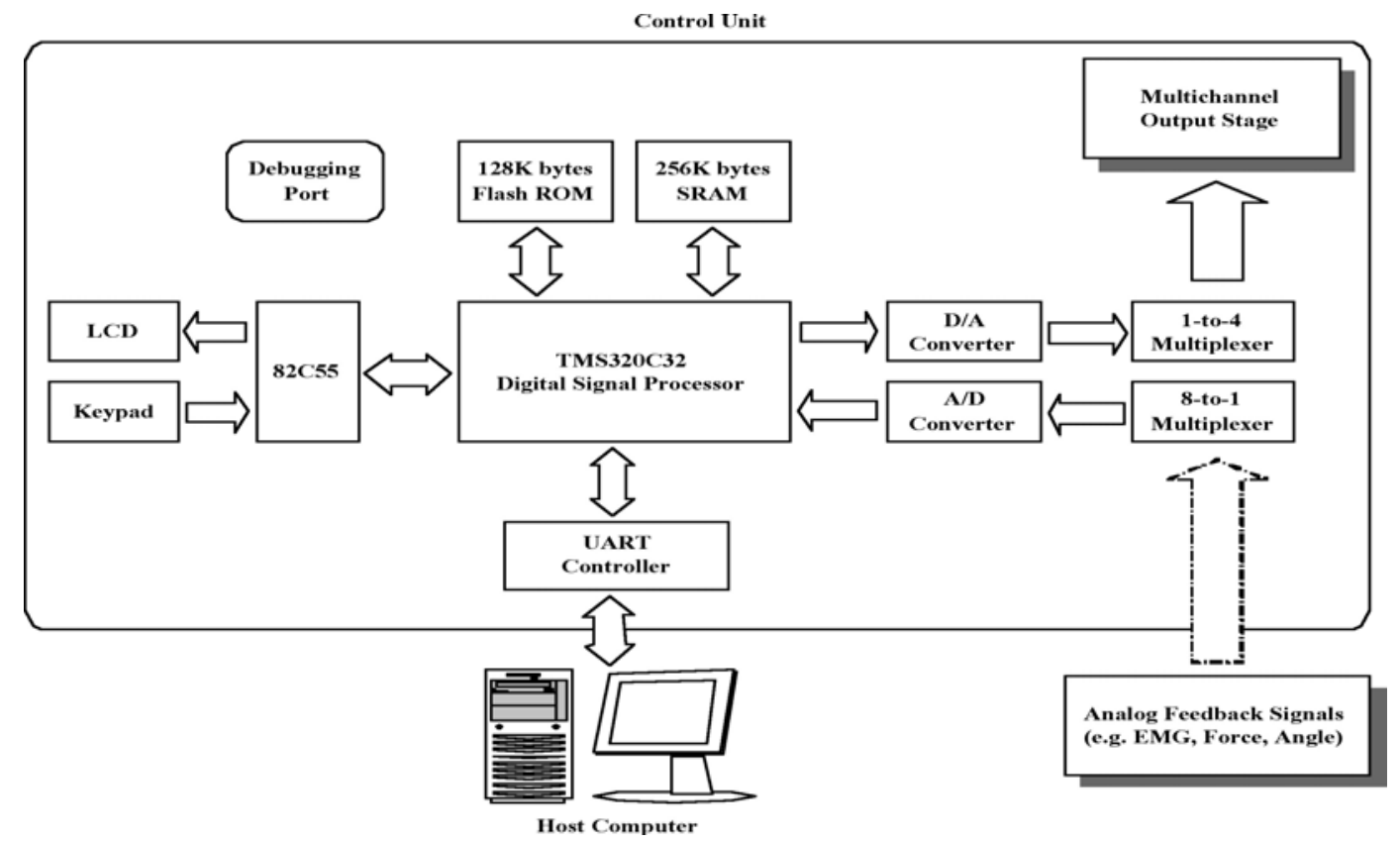

Fig. 1. Block diagram of our electrical stimulator.
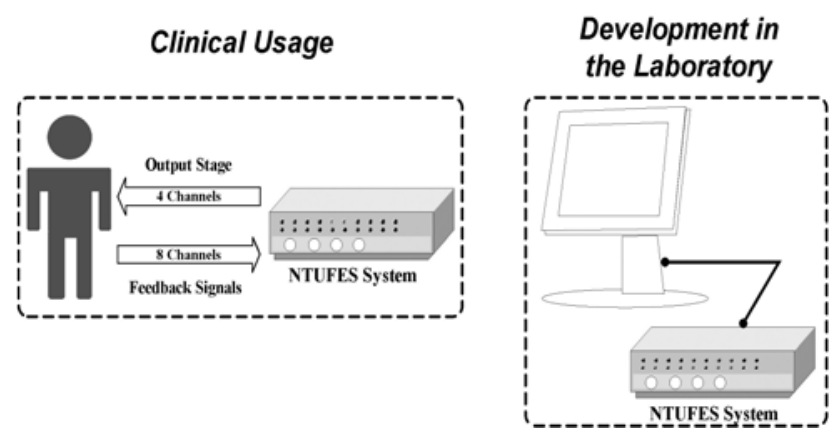

Fig. 2. Concepts of the NTUFES system: from the viewpoint of clinical usage and development in the laboratory.

decreased dramatically. To solve this problem, a battery-array is usually used to supply a high voltage source, but the entire system may be very heavy and expensive, and most importantly, not be easily portable. Therefore, to implement the driving stage, a modified constant-current circuit, as depicted in Fig. 3(a), has been proposed. Some features of this circuit are: (1) The output current $\boldsymbol{I}_{\text {in }}$ can be simply adjusted by $\boldsymbol{R}_{1}$ and is linearly proportional to the input voltage $\boldsymbol{V}_{\text {in }}$

$$
I_{\text {in }}=\frac{V_{\text {in }}}{R_{1}} .
$$

Therefore, the stimulation current $\boldsymbol{I}_{0}$ can be determined by the following equation

$$
I_{o}=\frac{I_{i n}}{10} .
$$

(2) The noise cancellation design ensures that there is no current output when initializing the electrical stimulator (as depicted in Fig. 3(b)). (3) The NTUFES system is isolated via the isolated transformers and powered by $9 \mathrm{~V}$ alkaline batteries. In addition, the chassis of the stimulator is hardwired to the ground. These are all electrical safety considerations.

\subsection{Innovative User-friendly Toolbox for the Electrical Stimulator}

In order to ease the operation of the electrical stimulator for physicians and clinical users, a userfriendly interface is necessary. Therefore, we have chosen to use LabVIEW, which is a graphical objectoriented program that is easier for the clinical practitioners and beginners to use than the conventional text-based programming languages (for instance, assembly or $\mathrm{C}$ languages), to implement the man-machine interface for the NTUFES system. The basic entity of the LabVIEW program is the virtual instrument (VI) using a graphical presentation. Users 


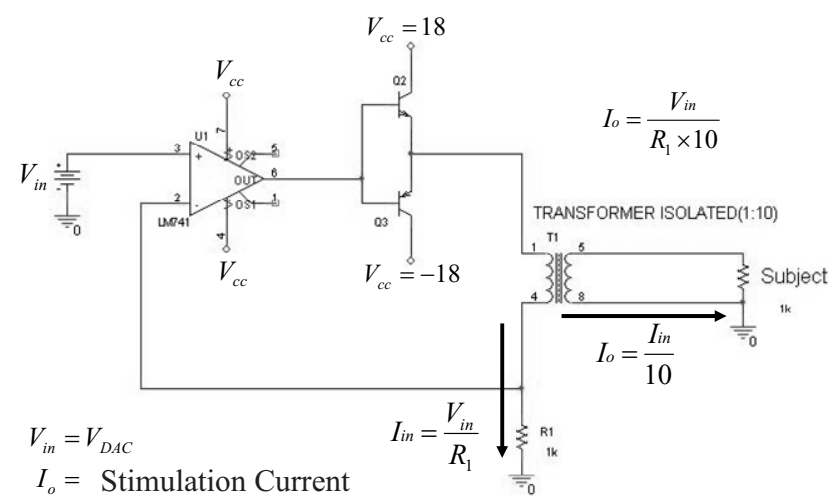

Fig. 3(a). The modified constant-current circuit.

can design the interfaces for their studies just by using the mouse to drag and drop the VIs. In the NTUFES system scheme, the electrical stimulator can be controlled by a host computer via the RS-232 interface with a proprietary protocol. We have designed several essential VIs using this protocol (please see Table I).

These essential VIs are as follows, "DSPinitiate.vi", "Stimulator-RS232.vi", "CloseStimulator.vi", and "Get-Currents.vi", respectively. These VIs can be put into a toolbox library, and users

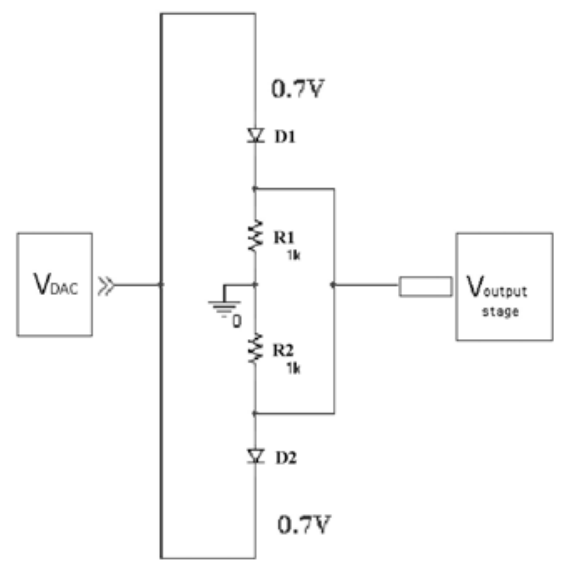

Fig. 3(b). Noise cancellation circuit of the electrical stimulator.

can just drag and drop them, using the mouse, to design their own man-machine interfaces for the experiment quickly and easily. The contents of "DSPinitiate.vi" include sending bytes to the DSP to start the electrical stimulator, defining the serial port number, and checking the communication between the host computer and the NTUFES system. Fig. 4 illustrates

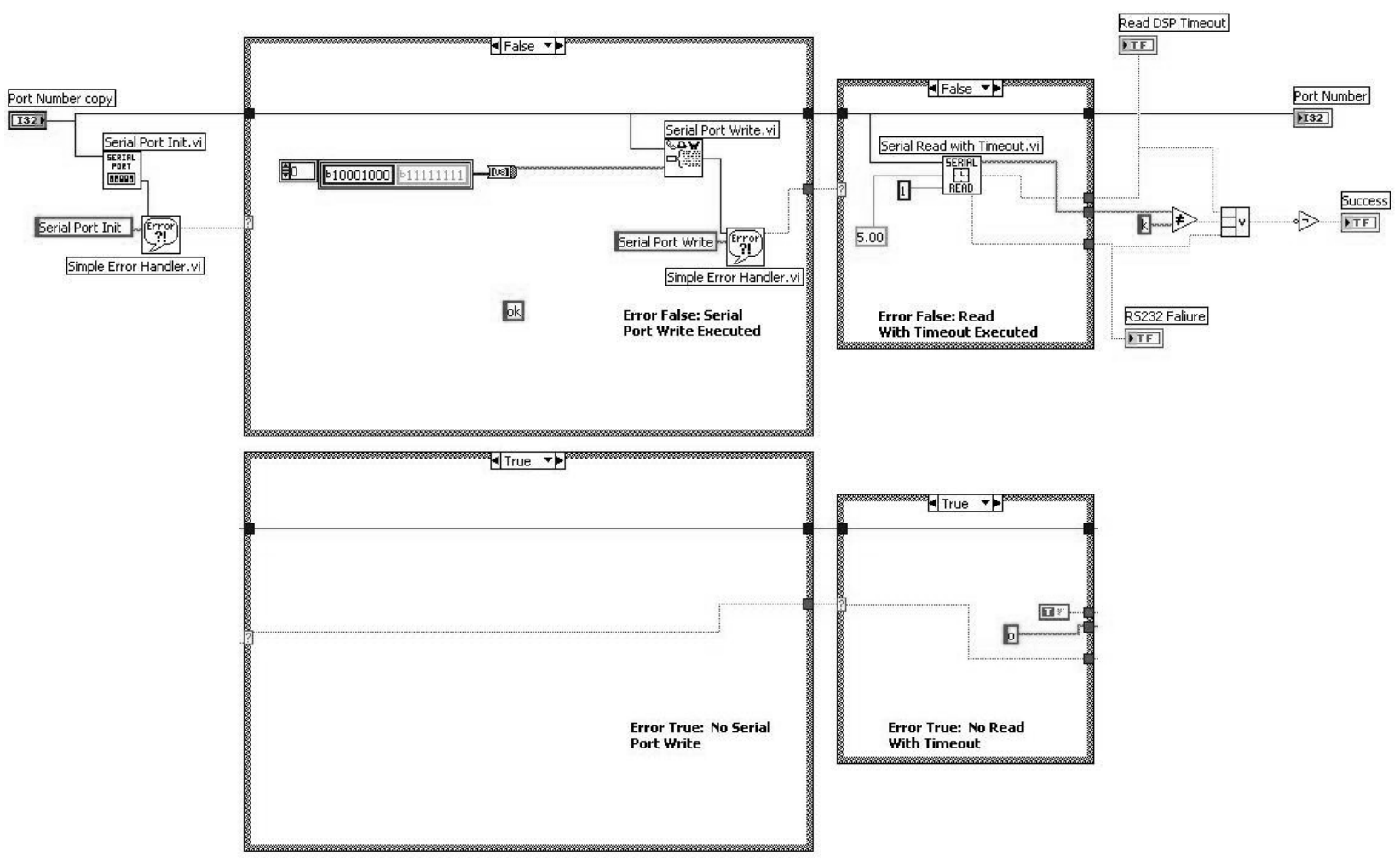

Fig. 4. The block diagram of "DSP-initiate.vi". 
the details of "DSP-initiate.vi". The contents of "Stimulator-RS232.vi" include rechecking the communication between the NTUFES system and the host computer, and defining the stimulation parameters, including channel numbers, current, pulsewidth, stimulation patterns, frequency, and stimulation time. The details of "Stimulator-RS232.vi" are illustrated in Fig. 5. The contents of "CloseStimulator.vi" include checking the serial port number and sending bytes to the DSP to stop the electrical stimulator. In addition, the real-time currents or other feedback signals from the electrical stimulator can be plotted to the monitor of the host computer through "Get-Currents.vi". Fig. 6 shows the front panel and block diagram of "Get-Currents.vi". By having these
Table I. The protocol used in the electrical stimulator

\begin{tabular}{lll}
\hline \hline Start: start the computer mode & $=>10001000$ & $\rightarrow 0 \times 88$ \\
\hline Packet $0:$ start system & $=>10000001$ & $\rightarrow 0 \times 81$ \\
Packet 1: channel + pattern + mod & $=>0$ aabbccc & \\
Packet 2: pulse-width & $=>0$ aaaaaaa & \\
Packet 3: current & $=>0$ aaaaaaa & \\
Packet 4: offset & $=>0$ aaaaaaa & \\
Packet 5: frequency & $=>0$ aaaaaaa & \\
Packet 6: stimulation time & $=>0$ aaaaaaa & \\
Packet 7: end and standby & $=>10000000$ & $\rightarrow 0 \times 80$ \\
\hline Stop: stop the computer mode & $=>11111111$ & $\rightarrow 0 \times F F$ \\
\hline \hline
\end{tabular}

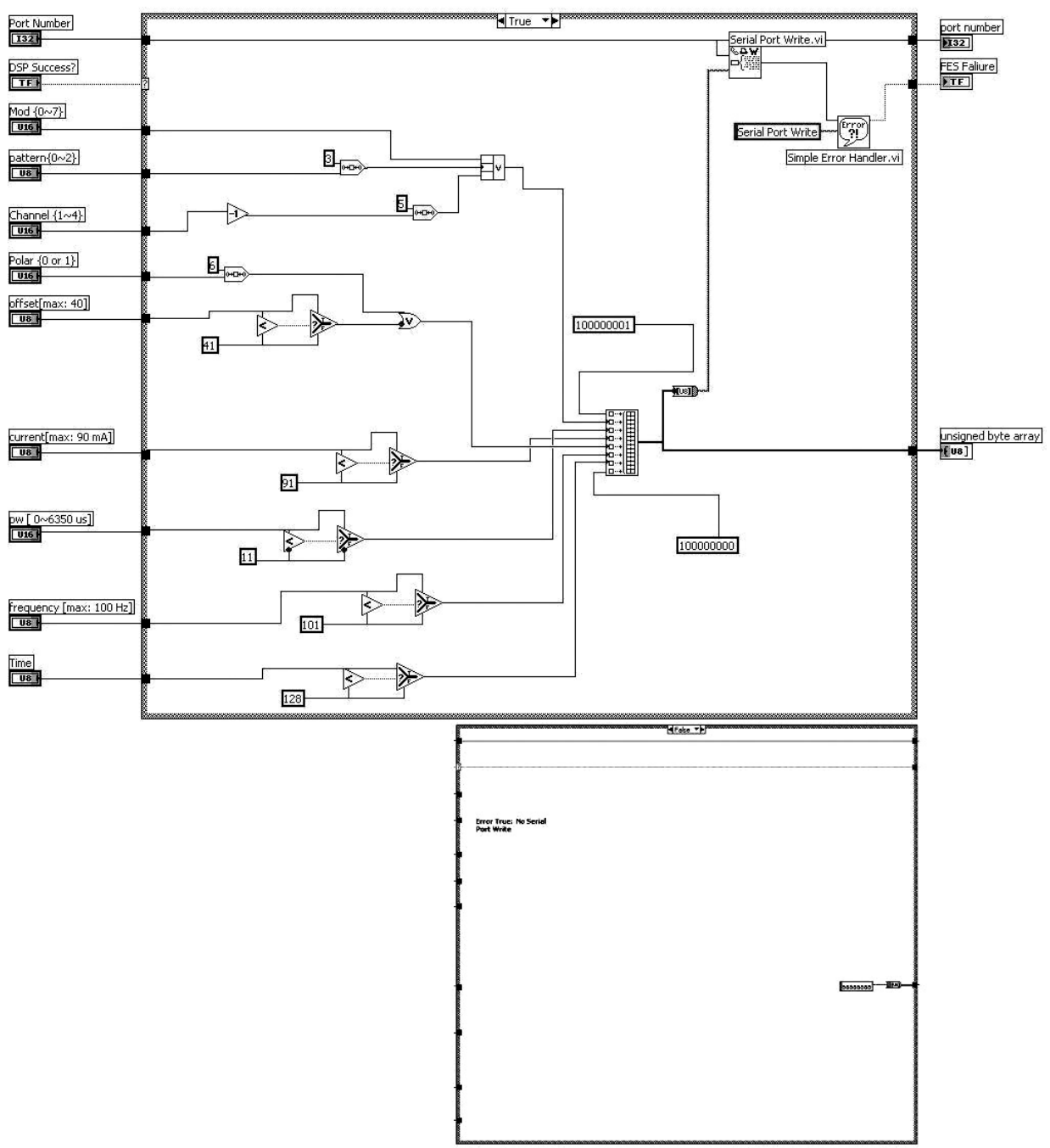

Fig. 5. The block diagram of "Stimulator-RS232.vi". 

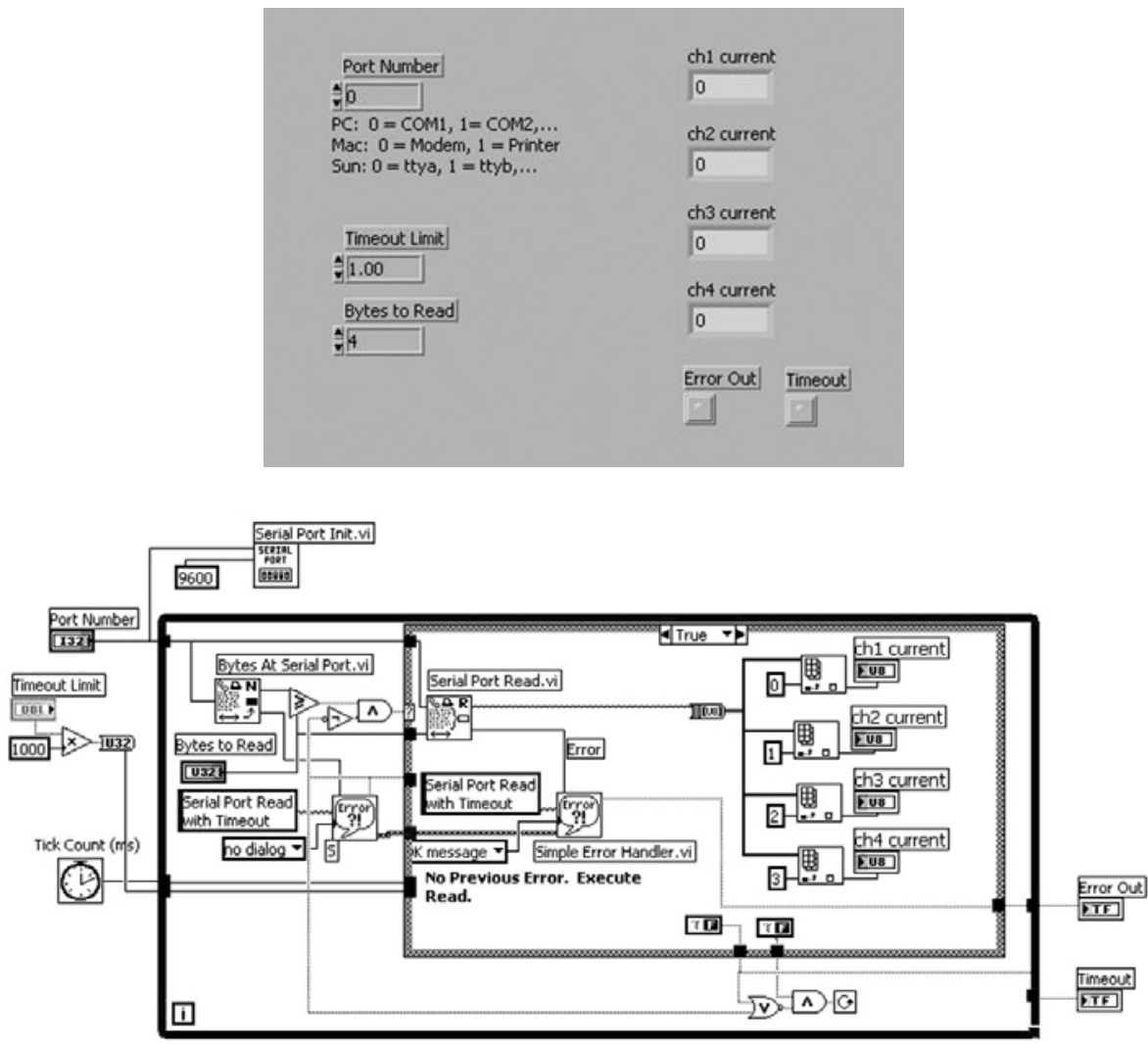

Fig. 6. "Get-Currents.vi": (a) the front panel, and (b) the block diagram.

VIs in the toolbox, we can design the visual instrument for the NTUFES system easily and conveniently. The following sections include the results and a discussion of the proposed design for the electrical stimulator.

\section{RESULT}

The proposed electrical stimulator with its versatile LabVIEW-based toolbox is presented in this article. The specifications of the NTUFES system are summarized in Table II. This system is versatile and feasible from the viewpoint of the hardware and software designs. In addition, the electrical stimulator has been evaluated by many groups in Taiwan, in the areas of shoulder joint control, the pedaling wheelchair system, muscle profile analysis, gait pattern training, and gastric function restoration. Moreover, the user can program his man-machine interface easily with the versatile LabVIEW-based toolbox, or by utilizing the GUI to set up stimulation parameters for the experiment. Users can also start the stimulation channels simultaneously or individually, according to the conditions of their studies. Fig. 7 demonstrates the man-machine interface using this toolbox in a hand function restoration experiment [27-28], and it can be used with multiple sequences in another electrical stimulation experiment, too. From these demonstrations, it can be seen that the proposed FES system, combined with its user-friendly toolbox, can surely provide clinical researchers a good environment for their studies, and that this design will cooperate with the system to restore functions successfully.

\section{DISCUSSION AND CONCLUSION}

The paper describes a versatile toolbox design for the electrical stimulator. Using this toolbox, the manmachine interface can be easily implemented in a LabVIEW-based environment. The NTUFES system, with its powerful DSP-based kernel, multi-channel stimulation, and programmable design, is illustrated herein. With great flexibility, the system can synthesize the stimulation patterns and waveforms using the element-envelope method.

We have designed a user-friendly toolbox especially for clinical users. Traditionally, physicians 


\section{Table II. Specifications of the NTUFES system}

\begin{tabular}{ll}
\hline \hline Number of Channels & 4 \\
Output Mode & Constant-Current \\
Current Output & $0 \sim 90 \mathrm{~mA}$ \\
Stimulation Patterns & Biphasic, Rectangle, and Triangle \\
Time Resolution & $50 \mathrm{~m}$ \\
Pulse-width & $50-6350 \mathrm{~ms}$ \\
Frequency Range & $1-100 \mathrm{~Hz}$ \\
Stimulation Time & $0-9999 \mathrm{~s}$ \\
Data Link with Host PC & RS-232, 9600 bps \\
OS Platform & Windows $98 / \mathrm{Me} / \mathrm{NT} / 2000 / \mathrm{XP}$ \\
Man-Machine Interface & LabVIEW-based environment \\
\hline \hline
\end{tabular}

have used electrical stimulators for clinical research, but they might not be familiar with the $\mathrm{C}$ languages to further design their own experiments. For that reason, we have developed a FES system with a user-friendly man-machine interface design in a LabVIEW-based environment. LabVIEW consists of executable codes controlled via a graphical front panel on the screen, similar to a real instrument. In contrast to the conventional text-based programming languages, LabVIEW is programmed on the basis of front panel elements and block diagrams. Behind the front panel of an instrument is an assembly of electronic modules that performs the desired function. In addition, the front panel specifies the inputs called controls, and the outputs, called indicators. Users can employ a variety of controls and indicators, such as knobs, switches, buttons, and graphs, to create an easily understandable virtual operating environment; they can simply connect all these elements by means of a wiring tool. Each front panel has a corresponding block diagram, which defines the actual data flow between the inputs and outputs. The above-mentioned are the basic concepts of the LabVIEW-based environment, and for clinical users, such as physicians and physical therapists, using LabVIEW is surely easier and quicker than using conventional text-based programming languages.

In other studies, LabVIEW has also been used for its powerful alternatives to scientific and biomedical engineering programming [29-31]. In our study, to make the electrical stimulator more flexible and friendly, we have written the communication protocols

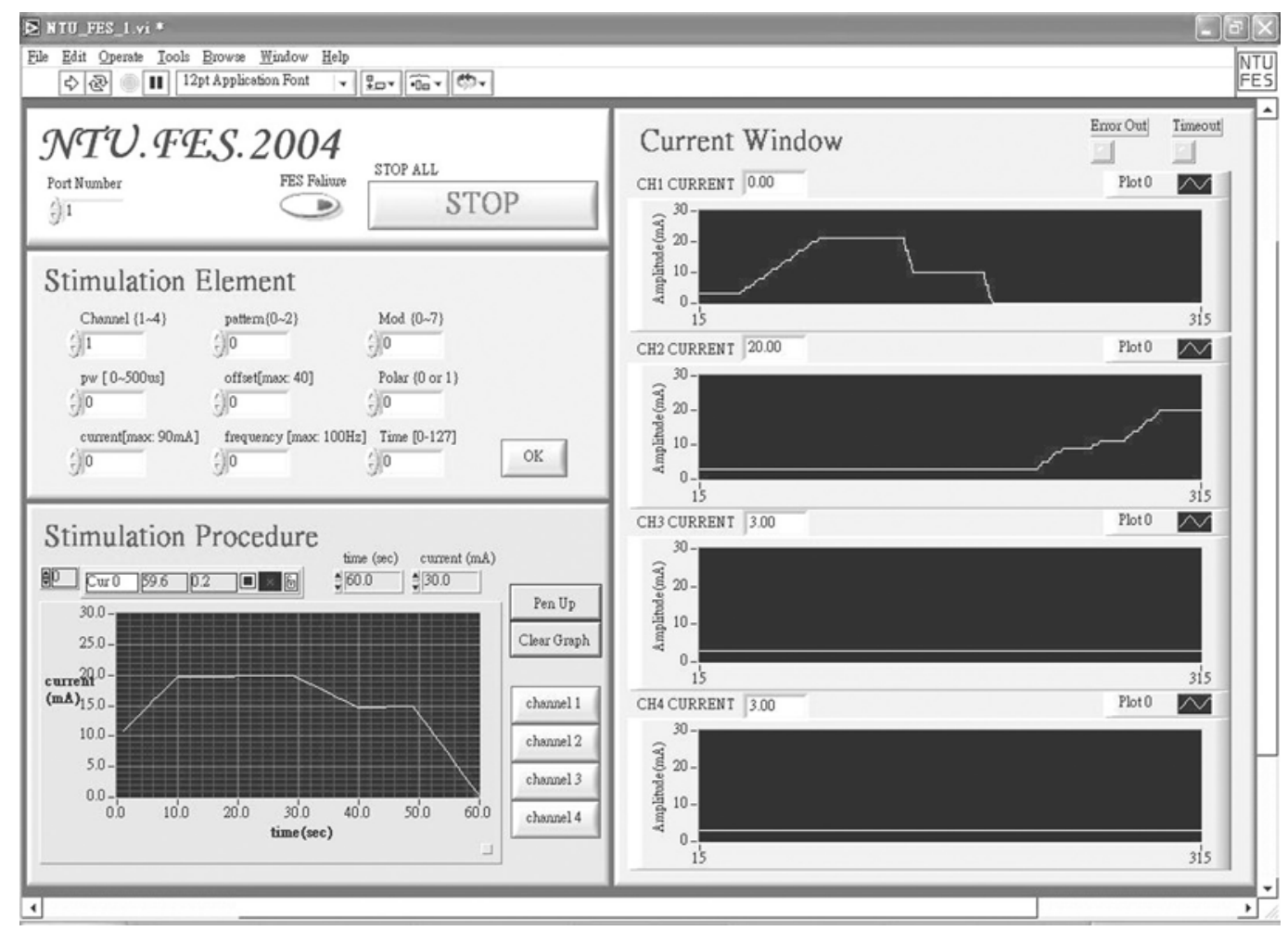

Fig. 7. The man-machine interface with 4-channel stimulations and adjusted parameters. 
for the stimulation parameters. A host computer can directly control the stimulator via a standard RS-232 interface. The users can easily adjust the stimulation parameters, such as the currents, pulse-width, frequency, stimulation patterns, and total stimulation times. Moreover, the NTUFES system can record the feedback signals of the ADCs, and with suitable control algorithms, a close-loop FES system can be implemented easily.

In summary, the NTUFES system can be considered a versatile and full-featured stimulator for various applications, with its high flexibility in stimulation patterns and multi-channel designs. Most importantly, this system can be tuned by parameters, such as waveform types, currents, stimulation time, etc, in its man-machine interface so that suitable electrical stimulation can be generated. This electrical stimulator can bring great benefit to clinical users, and will be very helpful when they design experiments. The procedure described above can also be implemented for different research purposes.

\section{REFERENCE}

1. Phillips CA: Functional electrical rehabilitation: technological restoration after spinal cord injury. In: Springer-Verlag, New York, 1991.

2. Reilly JP: Applied bioelectricity: from electrical stimulations to electropathology. In: SpringerVerlag, New York, 1998.

3. Liberson WT, Holmquest HJ, Scott D, and Dow A: Functional electrotherapy: stimulation of the personal nerve synchronized with the swing phase of the gait of hemiplegic patients. Arch Phys Med Rehab 1961; 42: 101-105.

4. Abbas JJ: Feedback control of coronal plane hip angle in paraplegic subjects using functional neuromuscular stimulation. IEEE Trans Biomed Eng 1991; 38: 687-698.

5. Bernotas LA, Crago PE, and Chizeck HJ: A discrete-time model of electrically stimulated muscle. IEEE Trans Biomed Eng 1986; 33: 829938.

6. Crago PE, Lan N, Veltink PH, Abbas JJ, and Kantor C: New control strategies for neuroprosthetic systems. J Rehab Res Dev 1996; 33: 158-172.

7. Peckham PH, Marsolais EB, and Mortimer JT: Restoration of key grip and release in the C6 tetraplegic patient through functional electrical stimulation. J Hand Surg 1980; 5: 462-469.

8. Alon G, Dar A, Katz-Behiri D, Weingarden H, and Nathan R: Efficacy of a hybrid upper limb neuromuscular electrical stimulation system in lessening selected impairments and dysfunctions consequent to cerebral damage. J Neur Rehab 1998; 12: 73-80.

9. Gad A and Keith M: Persons with C5 or C6 tetrapleagia achieve selected functional gains using a neuroprosthesis. Arch Phys Med Rehab 2003; 84: 119-124.

10. Wijman CA, Stroh KC, Van Doren CL, Thrope GB, Peckham PH, and Keith MW: Functional evaluation of quadriplegic patients using a hand neuroprosthesis. Arch Phys Med Rehab 1990; 71: 1053-1057.

11. Valencic V, Vodovnik L, Stefancic M, and Jelnikar T: Improved motor response due to chronic electrical stimulation of denervated tibialis anterior muscle in humans. Muscle Nerve 1986; 9: 612-617.

12. Gordon $T$ and Mao J: Muscle atrophy and procedures for training after spinal cord injury. Phys Ther 1994; 74: 50-60.

13. Baldi JC, Jackson RD, Moraille R, and Mysiw WJ: Muscle atrophy is prevented in patients with acute spinal cord injury using functional electrical stimulation. Spinal Cord 1998; 36: 463-469.

14. Thrope GB, Peckham PH, and Crago PE: A computer-controlled multichannel stimulation system for laboratory use in functional neuromuscular stimulation. IEEE Trans Biomed Eng 1985; 32: 363-370.

15. Buckett JR, Peckham PH, Thrope GB, Braswell SD, and Keith MW: A flexible, portable system for neuromuscular stimulation in the paralyzed upper extremity. IEEE Trans Biomed Eng 1988; 35: 897904.

16. Meadows P: Portable electrical stimulation systems. IEEE Aero El Sys Mag 1991; 6: 6-10.

17. Ilic M, Vasiljevic D, and Popovic DB: A programmable electronic stimulator for FES systems. IEEE Trans Rehab Eng 1994; 2: 234-238.

18. Cheever EA, Thompson DR, Cmolik BL, Santamore WP, and George DT: A versatile microprocessor-based multichannel stimulator for skeletal muscle cardiac assist. IEEE Trans Biomed Eng 1998; 45: 56-67.

19. Chen YL, Chang WH, Chen SC, Sheu PF, and Chen WL: The development of a knee locker with closed-loop functional electrical stimulation (FES) for hemiplegia in gait training. Disabil Rehab 2003; 25: 916-921.

20. Chang GC, Luh JJ, Laio GD, Lai JS, Cheng CK, Kuo BL, and Kuo TS: A neuro-control system for the knee joint position control with quadriceps stimulation. IEEE Trans Rehab Eng 1997; 5: 2-11.

21. Crago PE, Nakai RJ, and Chizeck HJ: Feedback regulation of hand grasp opening and contact force during stimulation of paralyzed muscle. IEEE Trans Biomed Eng 1991; 38: 17-28.

22. Grill JH and Peckham PH: Functional 
neuromuscular stimulation for combined control of elbow extension and hand grasp in C5 and C6 quadriplegics. IEEE Trans Rehab Eng 1998; 6: 190-199.

23. Kilgore KL, Peckham PH, Thrope GB, Keith MW, and Stone KAG: Synthesis of hand grasp using functional neuromuscular stimulation. IEEE Trans Biomed Eng 1989; 36: 761-769.

24. Nathan RH and Ohry A: Upper limb functions regained in quadriplegia: a hybrid computerized neuromuscular stimulation system. Arch Phys Med Rehab 1990; 71: 415-421.

25. Popovic MR, Curt A, Keller T, and Dietz V: Functional electrical stimulation for grasping and walking: indications and limitations. Spinal Cord 2001; 39: 403-412.

26. Wu HC, Young ST, and Kuo TS: A versatile multichannel direct-synthesized electrical stimulator for FES applications. IEEE Trans Instrum Meas 2002; 51: 2-9.

27. Chiou YH, Chen SC, Lai JS, and Kuo TS: A noninvasive functional electrical stimulation system with patient-driven loop for hand function restoration. Proceedings of the 26th Annual International Conference of the IEEE Engineering in Medicine and Biology Society 2004; 1: 41304132.

28. Chiou YH, Luh JJ, Chen SC, Lai JS, and Kuo TS: Optimal strategy of the patient-driven command controller for restoring hand functions by functional electrical stimulation. Proceedings of the 25th Annual International Conference of the IEEE Engineering in Medicine and Biology Society 2003; 1: 1551-1553.

29. Moore JH: Artificial intelligence programming with LabVIEW: genetic algorithms for instrumentation control and optimization. Comput Meth Programs Biomed 1995; 47: 73-79.

30. Poindessault JP, Beauquin C, and Gaillard F: Stimulation, data acquisition, spike detection and time/rate analysis with a graphical programming system: an application to vision studies. J Neurosci Meth 1995; 59: 225-235.

31. Nordstrom MA, Mapletoft EA, and Miles TS: Spike-train acquisition, analysis and real-time experimental control using a graphical programming language (LabView). J Neurosci Meth 1995; 62: 93-102. 